\title{
Miranda
}

Revue pluridisciplinaire du monde anglophone /

Multidisciplinary peer-reviewed journal on the English-

speaking world

$12 \mid 2016$

Mapping gender. Old images ; new figures

\section{Margaret Thatcher : une avancée pour les femmes britanniques?}

\author{
Karine Rivière-De Franco
}

URL : http://journals.openedition.org/miranda/8695

DOI : 10.4000/miranda.8695

ISSN : 2108-6559

Éditeur

Université Toulouse - Jean Jaurès

Référence électronique

Karine Rivière-De Franco, " Margaret Thatcher : une avancée pour les femmes britanniques ? 》, Miranda [En ligne], 12 | 2016, mis en ligne le 02 mars 2016, consulté le 16 février 2021. URL : http:// journals.openedition.org/miranda/8695; DOI : https://doi.org/10.4000/miranda.8695

Ce document a été généré automatiquement le 16 février 2021.

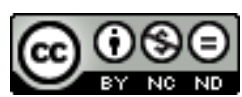

Miranda is licensed under a Creative Commons Attribution-NonCommercial-NoDerivatives 4.0 International License. 


\title{
Margaret Thatcher : une avancée pour les femmes britanniques?
}

\author{
Karine Rivière-De Franco
}

\section{Introduction}

1 Si la Grande-Bretagne est habituée aux souveraines - de Mary I à Elizabeth II, en passant par Elizabeth I et Victoria -, en revanche, concernant la fonction de Premier ministre, et notamment depuis que ce poste est investi du plein pouvoir politique, les femmes s'illustrent par leur absence. Margaret Thatcher, élue député en 1959, leader du Parti conservateur en 1975 et Premier ministre pendant onze ans, de 1979 à 1990, apparait comme une femme au parcours exceptionnel dans l'histoire politique britannique. Quelques données permettent de prendre la pleine mesure de son ascension politique. A sa naissance, seules les femmes de plus de trente ans disposent $\mathrm{du}$ droit de vote (Representation of the People Act, 1918) ${ }^{1}$ et la participation des femmes à la vie politique ne se fait que très progressivement. Les femmes ne représentent que $4 \%$ des parlementaires lorsque Margaret Thatcher entre à la Chambre des communes en 1959 et $9 \%$ lors du scrutin qui suit sa démission ${ }^{2}$. L'importance de leur fonction et l'éventail de leur position au sein des partis ou du gouvernement ne s'accroissent que très graduellement et ce n'est que plusieurs années après qu'elle ait été remplacée que des femmes sont nommées à la tête de ministères qui ne sont pas traditionnellement confiés à des femmes ${ }^{3}$. A ce jour, aucune femme n'a occupé la fonction de Ministre des finances britannique et Margaret Thatcher reste la seule à avoir dirigé l'un des trois principaux partis politiques ${ }^{4}$. Dans les années 1970-1980, la sphère politique britannique constitue, malgré la présence de Barbara Castle ou de Shirley Williams, un univers fortement masculin et, dans ces circonstances, Margaret Thatcher, elle-même, ne pouvait pas imaginer occuper un jour les plus hautes fonctions politiques. Dans un entretien accordé au Liverpool Daily Post le 17 juin 1974, soit moins d'un an avant d'être élue à la tête du Parti conservateur, elle déclarait : « Il faudra des années avant qu'une femme devienne leader du parti ou Premier ministre. Je ne crois pas que cela arrive de mon vivant $»^{5}$. 
2 L'ascension politique de Margaret Thatcher a suscité nombre de débats et de réactions, sur les questions de masculinité et de féminité et de sphères publique et privée. A la fois source d'espoirs pour certaines féministes et cible aisée pour l'élite politique sur le territoire duquel elle empiétait, son positionnement en tant que femme politique a soulevé des interrogations nouvelles et a donné lieu à des commentaires divergents, parfois admiratifs, parfois critiques. Toutes les ambiguïtés et les paradoxes se trouvent résumés dans le surnom que le journal de l'Armée Rouge, le Red Star, lui donna pour la première fois en 1976 : la Dame de Fer. Par ailleurs, membre de la Nouvelle droite, elle s'est définie elle-même comme une " responsable politique de conviction $»^{6}$. Sa manière de diriger s'est avérée bien éloignée de l'image traditionnelle de la femme de l'époque et sa conception autoritaire du pouvoir correspondait, selon Theodore Millon, à un modèle de personnalité spécifique : «le Dominant » (Steinberg 238-245). La classe politique, en majorité issue de la bourgeoisie anglaise et éduquée entre hommes dans les prestigieuses public schools, s'est vue contrainte de s'adapter à une femme qui bouleversait leurs repères classiques. Certains ministres ont avoué leur irritation d'être commandés par une femme, notamment lorsqu'ils étaient humiliés en public ${ }^{7}$. Elle n'a pas hésité à écarter ceux qui ne lui témoignaient pas d'un soutien sans faille, notamment ceux qu'elle qualifiait de " timorés $»^{8}$, qu'elle opposait aux « purs et durs ». Margaret Thatcher, cette "fille d'épicier " comme l'Allemand Helmut Schmidt et le Français Valéry Giscard d'Estaing la surnommaient fit souvent l'objet d'une représentation manichéenne et caricaturale, comme les réactions à l'annonce de son décès le 8 avril 2013 l'ont, à nouveau, illustré.

3 Cet article vise à analyser les déclarations et les actes de cette femme au parcours hors du commun sous un angle spécifique, celui des «enjeux féminins »; il s'agit d'étudier son positionnement par rapport aux sujets qui concernent en premier lieu les femmes, que ce soit pour des raisons biologiques, comme la contraception ou certaines maladies, ou pour des motifs sociaux, comme la parité ou la garde d'enfants, pour reprendre la définition établie par Joni Lovenduski et Azza Karam $(1998,5)$, autrement dit, les politiques dont les répercussions directes et immédiates touchent davantage cette catégorie de population, pour citer Linda Trimble et Jane Arscott $(2003,12)$. La défense d'intérêts qui seraient spécifiquement féminins constitue, en effet, l'un des motifs fréquemment avancés par ceux et celles qui militent pour une représentation politique féminine supérieure. Cet argumentaire repose sur la corrélation supposée entre la représentation descriptive (RD) et la représentation substantive (RS) : ce n'est que si le Parlement et le gouvernement comprennent davantage de femmes (RD ou numérique) qu'elles pourront influencer les débats et défendre au mieux les intérêts de l'ensemble de la population féminine (RS) (Childs 2008). Dans le cas présent, il ne s'agit pas tant d'une question de nombre - les femmes représentant moins de $10 \%$ des parlementaires à l'époque -, mais d'une position d'autorité : l'influence d'une femme à la tête du pouvoir politique, chargée de décider de la législation à soumettre au Parlement, au sein duquel le Parti conservateur disposait d'une nette majorité entre 1979 et $1990^{\circ}$.

Comment peut-on qualifier l'impact de Margaret Thatcher pour les femmes britanniques ? Quel intérêt a-t-elle exprimé, à travers ses paroles et ses actes, pour les " enjeux féminins »? Si certaines se sont réjouies à la perspective qu'une femme dirige le pays, leurs espoirs n'ont-ils pas été déçus ? De nombreux ouvrages ont été publiés sur Margaret Hilda Roberts, sur sa manière de diriger le Parti conservateur et de gouverner le pays (Kavanagh 1989, Young 1990, Leruez 1991...). Des études ont également été 
menées dans le but d'évaluer la corrélation entre le nombre de représentantes et leur comportement législatif, et notamment leur positionnement par rapport aux « enjeux féminins » (Campbell 2005, Childs 2002, 2004, Norris 1996, 2001, Bird 2005, Rivière-De Franco 2014...). Le présent article entend contribuer à la littérature existante à travers l'étude d'un vaste corpus de sources primaires, qui couvre la période au cours de laquelle Margaret Thatcher fut au pouvoir, de mai 1979 à novembre 1990. Ce corpus principal comprend l'intégralité des déclarations, des discours et des interviews du Premier ministre sur la période ${ }^{10}$; il est complété par les manifestes électoraux du Parti conservateur pour les scrutins de 1979, 1983 et $1987^{11}$, ainsi que par l'ensemble de la législation adoptée par le Parlement britannique durant ces 11 ans, 6 mois et 24 jours ${ }^{12}$. L'analyse, qui contient un volet quantitatif (fréquence des occurrences) et un volet qualitatif (étude de contenu), permet de déterminer dans quelle mesure la présence d'une femme au pouvoir s'est avérée bénéfique pour la population féminine britannique et de s'interroger sur les apparentes contradictions de l'ancien Premier ministre. Si Margaret Thatcher semble nier l'existence d'enjeux qui affecteraient plus particulièrement les femmes et s'oppose à toute forme de discrimination positive, de manière paradoxale, son discours, qui dépeint une vision classique de la femme et souligne des qualités spécifiquement féminines, tout comme l'utilisation de sa propre féminité dans sa communication politique, témoignent de la pertinence de ces questions. La thématique de la représentation politique permet d'explorer l'ensemble de ces ambiguïtés.

\section{Le refus de reconnaître toute spécificité liée au genre}

\section{Un manque d'intérêt pour les « enjeux féminins »}

5 L'analyse de la place accordée aux «enjeux féminins» par Margaret Thatcher représente un signe de son intérêt pour les préoccupations et les problèmes spécifiques rencontrés par ce sous-groupe de population. L'étude lexicologique des manifestes publiés par le Parti conservateur pour les élections législatives de 1979, 1983 et 1987 lorsque Margaret Thatcher était chef de parti - révèle que cette thématique est quasiment ignorée : une seule occurrence du terme «femme(s) » est relevée dans le programme de 1979, 4 dans celui de 1983 et 3 en 1987. En outre, l'analyse de ces occurrences en contexte fait apparaître que leur emploi se révèle, en majorité, générique, le terme étant inclus dans des expressions servant à désigner la population dans son ensemble; ainsi, 5 sur 8 sont associées au terme "homme(s)». Malgré une absence d'intérêt exprimé pour les « enjeux féminins » dans le programme électoral, reflet de la philosophie politique du Parti conservateur dans son ensemble, comment Margaret Thatcher s'est-elle positionnée personnellement?

6 L'exploitation de l'ensemble des déclarations, discours et interviews du Premier ministre, dans un premier temps sous la forme d'une recherche quantitative exhaustive, montre que parmi les 22674 documents de la période, 418 contiennent le terme « femme(s) », soit moins de $2 \%$. De plus, comme pour les manifestes, la majorité de ces occurrences ne fait pas directement et uniquement référence à la population féminine, mais constitue des synonymes pour les Britanniques ( les hommes et les femmes », «les hommes, les femmes et les enfants»). Seul un quart environ de ces 
occurrences (119 occurrences) traite de sujets qui préoccupent spécifiquement cette catégorie de population.

7 Une analyse de contenu permet de classer ces références en plusieurs catégories, même si certains documents abordent plusieurs thématiques. Le nombre le plus élevé d'occurrences porte sur les femmes et l'emploi (29), les femmes et la politique (19), les priorités et le bilan du gouvernement (15), les différences entre les hommes et les femmes (14), le parcours personnel de Margaret Thatcher (8), la question de la discrimination positive et le mouvement féministe (8). Une minorité concerne des références historiques (4), les thèmes de la santé (4), la famille (3) et l'éducation (3), ou sont des traits d'humour (4). La question des «enjeux féminins" ne représente clairement pas une priorité pour Margaret Thatcher, puisque lors de ses onze années de mandats, elle ne s'est exprimée sur ce sujet que dans $0,52 \%$ de ses déclarations, discours et interviews. Ces chiffres s'avèrent d'autant plus surprenants que nombre des occurrences relevées renvoient à des réponses à des questions de journalistes l'interrogeant sur son statut de première femme Premier ministre ; sans ces questions, qui l'obligent à aborder le sujet malgré elle, les chiffres seraient encore plus bas. A en juger par la fréquence numérique dans le discours politique, Margaret Thatcher ne semble pas s'intéresser à la population dont elle fait partie en termes de caractéristique sexuée, ou peut-être réfute-t-elle par là-même la question, les femmes n'étant pas, d'après elle, confrontées à des problématiques spécifiques. C'est ce qu'il ressort de certaines de ses déclarations, de manière plus ou moins explicite. Ainsi, lorsqu'un journaliste lui demande en 1986 si, en tant que femme Premier ministre, elle pense aux autres femmes lorsqu'elle prend des décisions politiques, elle répond par l'affirmative (" oui, tout à fait, tout à fait »), avant de ne citer qu'un seul exemple : son souci pour les veuves avec enfants, ce qui lui permet de conclure à l'importance de la famille ${ }^{13}$. L'année suivante, elle dévoile davantage sa pensée : interpellée sur la manière dont elle " aide » les femmes britanniques, elle estime que « les femmes s'aident elles-mêmes » et que le rôle du gouvernement se limite à créer des conditions favorables ${ }^{14}$. A la veille du scrutin de 1987, elle se montre encore plus explicite, indiquant : « Nous n'avons pas de politiques spécifiques pour les femmes. Les femmes ont désormais beaucoup plus de possibilités qu'auparavant. Je ne serais pas là sinon. Je souhaite simplement que davantage de femmes en profitent $»^{15}$. Elle indique, par ailleurs, qu'elle ne regrette pas de ne pas s'être adressée directement à l'électorat féminin dans le manifeste car la population ne doit pas être compartimentée ${ }^{16}$. Malgré une volonté affichée de ne pas reconnaître d'intérêts spécifiquement féminins, de manière concrète, l'action politique de Margaret Thatcher a-t-elle bénéficié aux femmes britanniques?

\section{Un maigre bilan en termes législatifs}

8 L'analyse de contenu révèle qu'une infime minorité des déclarations, discours et interviews du Premier ministre concerne l'action ou le bilan des gouvernements qu'elle a dirigés en termes d'« enjeux féminins » $(0,066 \%$ du corpus total). Margaret Thatcher se félicite néanmoins que la Grande-Bretagne se place en $2^{\text {ème }}$ position pour le taux d'activité des femmes au sein de la Communauté européenne, que le nombre de femmes ait augmenté dans l'enseignement supérieur ${ }^{17}$ ou que le Royaume-Uni soit le seul pays européen à avoir un taux de chômage des femmes inférieur à celui des hommes ${ }^{18}$. Toutefois, ce qui revient le plus fréquemment, c'est une mesure fiscale adoptée en fin de mandat: la taxation séparée des femmes mariées $^{19}$; cette réforme est présentée 
comme un «événement clé » et le Premier ministre se déclare «fière » d'appartenir à un gouvernement qui a adopté " cette mesure de justice envers les femmes, une mesure attendue depuis longtemps ", qui touche " 3 millions de femmes mariées » et qui contribue à «l'indépendance des femmes et à la protection de leur vie privée $»^{20}$.

De manière plus globale, l'analyse de l'ensemble de la législation adoptée par le Parlement britannique entre 1979 et 1990 montre que, sur les 581 lois votées ${ }^{21}, 20$ affectent, d'une manière ou d'une autre, les femmes dans des proportions plus importantes que les hommes $(3,44 \%$ du total). Sur les onze années de mandats de Margaret Thatcher, trois $(1979,1982$ et 1989) se caractérisent par l'absence de toute législation spécifique. Les 20 lois concernées portent, en majorité, sur les régimes matrimoniaux et familiaux. Trois se distinguent néanmoins : une loi sur la prostitution crée de nouvelles infractions pour les clients et augmente la durée maximale encourue pour tentative de viol (Sexual offences Act 1985), tandis qu'une autre concerne l'interdiction de l'excision (Prohibition of Female Circumcision Act 1985). Enfin, une loi sur la discrimination sexuelle (Sex Discrimination Act 1986) porte sur le travail des femmes en termes d'horaires et de périodes de travail ; toutefois, la Grande-Bretagne s'était vue contrainte de se mettre en conformité avec le droit européen ${ }^{22}$. Au final, Margaret Thatcher a mené peu d'action en faveur des femmes, et l'image que l'opinion publique retient de cette femme Premier ministre, c'est celle d'un leader à poigne, aux priorités plutôt masculines.

10 Ainsi, à l'opposé de l'image traditionnelle de la femme qui donne la vie, elle a engagé la Grande-Bretagne dans des conflits armés. Avec la victoire militaire contre l'Argentine aux Malouines en 1982, elle est devenue héros militaire, "paroxysme de la masculinité » (Thomson 216). Elle n'avait pas hésité deux ans auparavant à donner l'ordre d'attaquer lors de la prise d'otages à l'ambassade d'Iran à Londres. En Irlande du Nord, vêtue du béret rouge et du gilet pare-balles des régiments de parachutistes, elle s'est fait photographier dans un tank, véhicule qui renvoie à des " récits héroïques de courage masculin, de force et de fierté nationale stoïques " (Nunn 9-10) ${ }^{23}$. Elle n'a pas intercédé en faveur des activistes en grève de la faim emprisonnés en Irlande du Nord ; une dizaine d'entre eux mourront en prison. Le Premier ministre s'est également opposée aux femmes qui avaient établi un camp de la paix à Greenham Common dans le Berkshire afin de protester contre l'armement nucléaire. Ces dernières eurent recours à des images typiquement féminines, qu'elles opposèrent au discours scientifique et rationaliste du Premier ministre (Nunn 151). Enfin, le Premier ministre s'est déclarée pour le rétablissement de la peine de mort, ayant d'ailleurs voté contre son abolition (Thatcher 1993, 307). Combinés à une politique économique qui entraîne des taux de chômage record et l'augmentation des inégalités sociales, elle fut accusée par ses détracteurs, le Parti travailliste en tête, d'être une femme impitoyable et insensible. Margaret Thatcher affirme ne pas considérer les femmes comme une catégorie aux besoins spécifiques, et il est donc inutile d'adopter une législation particulière, les lois devant s'appliquer à tous et à toutes.

11 Certaines féministes se montrent encore plus critiques sur le bilan de Margaret Thatcher; selon elles, son indifférence pour la cause des femmes l'a conduite à mener des politiques aux répercussions, directes et indirectes, néfastes pour nombre d'entre elles. D'une part, les femmes furent davantage touchées que les hommes par certaines mesures, comme les réductions budgétaires dans le domaine de la santé, des transports ou du logement. D'autre part, certains projets remirent en cause leurs droits: le 
gouvernement tenta d'amender une loi qui garantissait le droit de retrouver son emploi après un congé maternité, les femmes furent considérées en recherche d'emploi uniquement si elles pouvaient justifier d'un mode de garde pour leur enfant et les allocations familiales furent réformées. Certaines déplorèrent que « le programme de la première femme Premier ministre semble, de manière ironique, heurter de plein fouet nombre des priorités des féministes" (Bashevkin 52) ${ }^{24}$, d'autres la qualifièrent de "Judas au féminin » pour avoir trahi la cause des femmes, et soulignèrent que «les questions de genre constituent le principal silence, l'absence majeure dans les convictions de Margaret Thatcher, auto-proclamée 'responsable politique de conviction'»(Webster 3) ${ }^{25}$. Pour le Premier ministre, les femmes ne constituent pas un groupe aux intérêts clairement différents du reste de la population, et elle se déclare farouchement opposée à toute forme de discrimination positive.

\section{Une opposition à toute forme de discrimination positive}

12 Margaret Thatcher se déclare contre toute forme de discrimination positive, quel que soit le domaine. Dans le milieu professionnel, les femmes doivent être en concurrence, selon les mêmes critères que les hommes et seuls les compétences et le mérite doivent être pris en compte. Lorsqu'elle s'adresse à des dirigeants d'entreprise en 1987, elle déclare ainsi : «Si une entreprise trouve une femme qui est très, très compétente, cette femme aura autant de chances d'évoluer, d'avoir une promotion que n'importe quelle autre personne avec ces compétences $»^{26}$. En théorie, la loi de 1970 (Equal Pay Act 1970) a effectivement mis fin aux inégalités de salaire et de conditions de travail entre les hommes et les femmes ; en réalité, les écarts de salaires (gender pay gap) restent importants, de l'ordre de $28 \%$ en 1980 et de $23 \%$ en 1990 (Perfect 8-9). Même si elle affirme ne pas souhaiter abolir la Equal Opportunity Commission, créée suite à la loi antidiscrimination de 1975 (Sex Discrimination Act), et chargée de lutter contre la discrimination sexuelle et de promouvoir l'égalité des $\operatorname{sexes}^{27}$, c'est surtout aux femmes elles-mêmes de faire leurs preuves: "La seule manière de lutter contre les préjugés, c'est que des femmes qualifiées et compétentes se présentent et fassent leur travail tout simplement $\aleph^{28}$. Nul besoin d'introduire de législation spécifique facilitant, par exemple, l'accès à certains secteurs ou de mener des campagnes d'information afin d'élargir le futur horizon professionnel des écolières.

Margaret Thatcher affirme d'ailleurs, à plusieurs reprises, que, personnellement, le genre ne constitue pas un critère pour estimer la valeur d'une personne :

Je juge quelqu'un d'après sa personnalité, cela ne dépend pas de si c'est un homme ou une femme, mais de si il/elle possède une personnalité suffisamment affirmée, un certain sens de la décision, et la capacité à affronter les problèmes au lieu de les fuir; c'est beaucoup plus important que de savoir si c'est un homme ou une femme $^{29}$

ou encore "la différence est une question de personnalité et non de sexe $»^{30}$, d'où le rejet de toute forme de quotas ou de mesures spécifiques en faveur des femmes.

Le Premier ministre se montre d'ailleurs très critique envers les femmes qui continuent à lutter pour l'égalité des sexes; elle estime que la «bataille a été globalement gagnée $»^{31}$ et elle dénonce « le ton strident » des militantes du mouvement de libération des femmes ${ }^{32}$. Lorsque, dans une émission télévisée pour enfants, un garçonnet 
l'interroge à ce sujet, elle répond qu'« elle n'aime pas trop » ce mouvement, qui n'est pas très féminin et qui ne se concentre pas sur l'essentiel; elle en profite pour réaffirmer sa croyance en la réussite au mérite : les femmes qui ont réussi n'ont pas demandé de traitement de faveur, elles ont démontré leurs qualités et leurs compétences $^{33}$. De même, quand elle s'adresse au congrès des femmes conservatrices en 1988, elle tourne en dérision le combat des féministes contre le sexisme de la langue anglaise lorsqu'elles s'attaquent à certains termes comme "chairman $»^{34}$. Malgré cette négation explicite des « enjeux féminins » et son opposition affichée à la discrimination positive, Margaret Thatcher développe d'autres arguments qui témoignent d'une certaine reconnaissance de ces mêmes spécificités.

\section{Une rhétorique qui révèle une dimension genrée}

\section{L'existence de compétences spécifiques}

Alors même que Margaret Thatcher déclare que les femmes doivent être traitées comme les hommes et qu'elles ne doivent pas bénéficier de traitement de faveur, dans nombre de déclarations, de discours ou d'interviews, elle leur attribue des qualités spécifiques, dont les hommes seraient dépourvus. Ainsi, le verbe «cope», qui renvoie à la capacité à faire face, à tenir le coup et à s'en sortir, est utilisé de manière récurrente pour décrire les femmes. Margaret Thatcher, tout comme sa mère, possédaient cette qualité35, comme elle l'explique: «quoi qu'il arrive, je prends ce qui arrive et je gère. Mais c'est ce que font la plupart des femmes de toute façon, et elles le font très bien $»^{36}$. Par ailleurs, les femmes sont, d'après elle, dotées d'un grand sens pratique ( « practical $) ; ;^{37}$ elles visent l'efficacité et privilégient l'action à la parole : «elles [les femmes] préfèrent agir plutôt que de faire de longs discours et j'ai remarqué, qu'au Parlement, elles se montrent très concrètes sur la manière de faire avancer les choses $»^{38}$. Le Premier ministre souligne le « bon sens » féminin, notamment lors d'une réunion internationale : « au sujet du Conseil européen, nos amis français ont été très, très difficiles et ce qu'ils ont fait manquait tellement de bon sens, et les femmes ont, elles, beaucoup de bon sens, à la différence des hommes ${ }^{39}$. Ces références au pragmatisme féminin se retrouvent à plusieurs reprises dans ses déclarations ${ }^{40}$. En outre, le parallèle entre les sphères politique et domestique est récurrent ${ }^{41}$, et les femmes seraient particulièrement compétentes pour diriger le pays, du fait de leur expérience de gestion du foyer. Lorsqu'un enfant l'interroge sur les similitudes entre son travail de Premier ministre et son rôle d'épouse et de mère, elle insiste sur la nécessité de ne pas vivre au-dessus de ses moyens et souligne les dangers du crédit ${ }^{42}$. Elle se vante d'ailleurs de ce qu'il a fallu attendre qu' « un gouvernement soit dirigé par une ménagère habituée à gérer sa famille pour que, pour la première fois en vingt ans, il équilibre ses comptes, et garde même une poire pour la soif $»^{43}$. Ce type de rhétorique, fondé sur le bon sens féminin, lui permet de rendre accessible des questions politiques qui peuvent paraître complexes ou rébarbatives pour une partie des Britanniques (Nunn 16). De manière paradoxale, cette vision du Premier ministre reflète une forme de sexisme inversé, cette dernière considérant les femmes, ou du moins certaines d'entre elles, comme supérieures aux hommes. Par ailleurs, aucun Premier ministre, y compris Margaret Thatcher, n'a, à ce jour, mis à profit cette supposée aptitude particulière en nommant une femme Ministre des finances. Plus généralement, si les femmes possèdent tant de qualités, pourquoi Margaret Thatcher ne s'est-elle entourée 
que d'hommes pour gouverner? Si elle affirme « vraiment apprécier travailler avec des femmes ", celles-ci occupent uniquement des fonctions subalternes, qu'elles soient secrétaires, femmes de ménage, ou responsables de sa garde-robe et de son courrier ${ }^{44}$. Le Premier ministre recrée une hiérarchie sociale sur le modèle des sphères séparées de l'époque victorienne. Les déclarations de Margaret Thatcher font ressortir ambiguïtés et contradictions : elle affirme qu'une personne doit être jugée selon sa personnalité, mais elle estime que certaines compétences sont liées au genre et elle tient également un discours assez traditionnel sur la place de la femme dans la société.

\section{Le rôle traditionnel dévolu aux femmes}

17 Le Premier ministre aborde la question de la place des femmes dans la société britannique sous deux aspects principaux : l'emploi et la famille, les deux étant fréquemment liés. En matière d'emploi, le thème du travail des femmes permet essentiellement au Premier ministre de justifier les chiffres du chômage. Ce type d'occurrences constitue près de $10 \%$ de l'ensemble des références faites aux femmes dans les discours, déclarations et interviews du Premier ministre. Margaret Thatcher explique que le chômage ne baisse pas, malgré l'augmentation du nombre d'emplois, car un nombre croissant de femmes mariées et de mères de famille travaillent ou souhaitent occuper un emploi ${ }^{45}$ comme elle l'affirme en février $1985:$ " toutefois, cette augmentation [du nombre d'emplois] ne s'est pas répercutée sur les chiffres du chômage car beaucoup de femmes mariées se sont mises à travailler $\aleph^{46}$. De manière implicite, ces femmes sont responsables des mauvais chiffres du chômage qui ont été reprochés à Margaret Thatcher tout au long de son mandat; sans elles, les «vrais " chômeurs (hommes) pourraient avoir retrouvé un emploi. Les 3 millions de chômeurs furent en effet atteints dans les années 1980, une augmentation spectaculaire comparée au 1 million des années 1970, toutefois difficilement imputables uniquement aux femmes désireuses d'avoir une occupation en dehors de leur foyer.

Le travail à temps partiel, second point abordé par le Premier ministre, est présenté comme une solution idéale qui permet aux femmes de combiner vie professionnelle et vie familiale. A aucun moment Margaret Thatcher n'envisage, de manière explicite, qu'il s'agisse d'une situation subie en raison de la rareté des emplois à temps complet et non d'un choix personnel. Elle déclare, par exemple, «qu'est-ce qu'il y a de mal à avoir un emploi à temps partiel ? Beaucoup de femmes aiment les temps partiels [...] Cela augmente le niveau de vie de leur famille et elles peuvent dépenser une partie de ce qu'elles ont gagné $»^{47}$, ou encore " cela [le travail à temps partiel] permet de s'occuper du foyer. Cela permet d'être à la maison avec les enfants une partie de la journée ${ }^{48}$. Elle défend même cette forme d'emploi contre la Commission européenne, qui y voit l'occasion de pratiques discriminatoires ${ }^{49}$.

Lorsqu'elle traite de la place de la femme dans la société britannique, Margaret Thatcher ne cesse de faire les éloges de la structure familiale traditionnelle et de souligner le rôle primordial qu'occupe la femme en son sein, notamment en tant que mère. Pour elle, c'est la garante de la « sauvegarde de l'institution que représente la famille $~^{50}$. Elle s'élève contre ce qu'elle considère comme la stigmatisation des mères au foyer qui préfèrent s'occuper de leur famille plutôt que de travailler et qui se sentent, d'après elle, culpabilisées par la société, renversant ainsi l'argumentaire classique $^{51}$. Elle reconnait à certaines mères l'envie de travailler, mais elle insiste, d'une 
part, sur l'importance du choix personnel (" c'est à elles de décider ») $)^{52}$, et, d'autre part, elle indique clairement que les enfants doivent toujours constituer la priorité ( $"$ je vous en supplie, ne faites jamais passer les enfants au second plan $»)^{53}$; en outre, les mères ne peuvent exercer une activité professionnelle qu'à condition d'avoir pris des « dispositions entièrement satisfaisantes " pour la garde de leurs enfants ${ }^{54}$. Comme l'analyse Susan Kingsley Kent, pour Margaret Thatcher, les femmes participent au rétablissement d'un ordre moral dans la société à travers le maintien de règles morales au sein du foyer et l'éducation des enfants (Kent 349). Son discours présente une vision traditionnelle de la femme, à l'opposé de son cas personnel, une femme moderne qui a réussi dans un monde d'hommes, tout en ayant une famille (Webster 101). Il paraît étonnant de la part de celle qui a toujours évolué dans des univers masculins, et a fait des choix professionnels atypiques pour une femme de l'époque, qu'il s'agisse de suivre des études scientifiques, des études de droit, de devenir la première présidente de l'Association des conservateurs de l'Université d'Oxford ou de travailler dans l'industrie. Comme elle le raconte lors d'un entretien télévisé en 1985, elle ne se considère pourtant pas comme une "outsider $\aleph^{55}$. En revanche, elle reconnaît qu'avec ses jumeaux Mark et Carol, il lui aurait été difficile d'avoir une telle carrière si elle n'avait pas été élue dans une circonscription proche de Londres et du Parlement, ce qui lui permettait de rentrer le soir ainsi que de préparer le petit-déjeuner de son mari ${ }^{56}$.

\section{Une féminité assumée}

20 En public, Margaret Thatcher affirme que la question du genre n'est pas pertinente dans l'exercice de ses fonctions, mais elle utilise pourtant sa féminité comme un argument politique qu'elle intègre à sa stratégie de communication. Même si elle a toujours veillé à être élégante et coquette, qu'elle le veuille ou non, la question de son apparence physique et vestimentaire prend davantage d'importance que si elle était un homme. Elle reconnait par exemple au sujet de la campagne électorale de 1987 : «Dans mon cas, les préparatifs pour l'élection impliquaient davantage que de la politique. Je devais également avoir la tenue adéquate " (Thatcher 1993, 575) ${ }^{57}$. En tant que femme politique, elle devait trouver un style conventionnel mais féminin, qui lui fournissait autorité et lui permettait de ne pas être accusée de renoncer à sa féminité, comme le décrit Wendy Webster (88). Elle s'entoura de conseillers, notamment Gordon Reece, qui lui firent abandonner le port de chapeaux, changer de coiffure, modifier sa façon de se maquiller ainsi que sa dentition. Parallèlement, sur les conseils de Tim Bell, un coach du National Theatre lui fit travailler sa voix.

21 Sur le plan de la communication, Margaret Thatcher se présenta comme une maitresse de maison modèle et parla de sa famille, de son rôle de mère et d'épouse, de son goût pour les vêtements ou pour le maquillage, allant jusqu'à révéler qu'elle achetait sa lingerie chez Marks \& Spencer. De nombreuses occasions photographiques la montrèrent en compagnie de ses enfants, en train de coudre, de poser du papier peint ou de faire ses courses; ces mises en scène permirent à la population féminine, notamment les mères au foyer et les ménagères - une section de l'électorat traditionnellement acquise aux conservateurs - de s'identifier au Premier ministre.

Enfin, l'analyse de ses discours révèle que Margaret Thatcher intègre son statut de femme à sa rhétorique, souvent sous forme de traits d'humour. Ainsi, lors du congrès annuel du Parti conservateur, elle compare les économies que doivent faire les 
entreprises aux régimes amincissants que les femmes connaissent bien ${ }^{58}$. Lorsque le journaliste David Frost lui demande s'il y aura, à nouveau un jour, un autre homme Premier ministre, elle répond avec humour « je pense qu'un jour, ils redeviendront à la mode ${ }^{59}$. Par ailleurs, lorsqu'elle rencontre d'autres femmes politiques aux hautes responsabilités, elle ne manque pas de souligner leur point commun, comme avec la présidente de l'Islande ${ }^{60}$ ou la Premier ministre yougoslave (" nous appartenons toutes deux au petit, mais éminent, cercle des femmes Premiers ministres ») ${ }^{61}$, et elle se compare à d'autres pionnières politiques, comme Golda Meir en Israël ${ }^{62}$.

Ses adversaires politiques travaillistes exploitent d'ailleurs cet argument contre elle. Lors des questions au Premier ministre aux Communes, Andrew Faulds s'étonne que " en tant que première femme Premier ministre", elle "introduise une législation sexiste et raciste qui ferait de nombreuses femmes des citoyennes de seconde zone ${ }^{63}$. Shirley Summerskill l'interpelle sur le même sujet en ayant recours au même argument : comment peut-elle trahir ainsi des femmes ${ }^{64}$ Helen McElhone dénonce la duplicité de sa campagne électorale de 1983, au cours de laquelle elle s'est présentée comme une mère et une ménagère ordinaires, alors que ses politiques font souffrir les femmes ${ }^{65}$. Winnie Ewing, du Parti national écossais, dénonce les fermetures de maternité s'adressant non pas au Premier ministre, mais à la mère et à la belle-mère ${ }^{66}$. Le paradoxe qui caractérise le positionnement de Margaret Thatcher quant à la féminité et aux "enjeux féminins»trouve son paroxysme dans la question de la représentation politique.

\section{La féminisation de la sphère politique}

Dotées de qualités spécifiques selon Margaret Thatcher, les femmes pourraient apporter une contribution originale à la sphère politique. Or, si le Premier ministre regrette qu'il y ait si peu de femmes politiques, elle ne propose aucun moyen de remédier à cette situation.

\section{Une volonté affichée de changement}

25 A travers ses déclarations, discours et interviews, Margaret Thatcher déplore le nombre peu élevé de femmes politiques; elle utilise notamment les termes de «déception » et de " peine " pour exprimer ses sentiments à ce sujet ${ }^{67}$. Chaque fois qu'un journaliste la questionne, elle répète son insatisfaction. Il est vrai qu'en 1979 elle faisait partie des 19 femmes députés (3\%) et que lorsqu'elle démissionna en novembre 1990, elles n'étaient que 41 (6\%). En 1986, elle fixe même des objectifs à atteindre en termes de représentation féminine : "nous voulons doubler, tripler, quadrupler ces chiffres. Fixons-nous d'abord l'objectif d'un tiers de femmes députés ${ }^{68}$; cet objectif semble encore lointain au $21^{\text {ème }}$ siècle (22\% après les élections de 2010$)$.

Elle explique pourquoi elle déplore la faible représentation numérique des femmes en n'avançant qu'une seule raison : les femmes politiques attireraient moins l'attention si elles étaient plus nombreuses (l'adjectif « conspicuous » est systématiquement employé). Elle déclare par exemple en 1987 : «tant que nous ne serons pas plus nombreuses à la Chambre des communes et au sommet de l'Etat, le seul fait d'être une femme retiendra toute l'attention ${ }^{69}$. Elle estime que les femmes politiques sont encore considérées comme une anomalie : plus nombreuses, «nous serions normales au lieu d'être 
considérées comme un phénomène $»^{70}$. Du début à la fin de son mandat, en GrandeBretagne comme à l'étranger ${ }^{71}$, en référence au Parlement de Westminster comme au Parlement européen ${ }^{72}$, elle n'envisage aucun autre motif afin d'encourager les femmes à se lancer en politique ${ }^{73}$. Elle ignore totalement d'autres arguments, plus tard cités par Anne Phillips dans son ouvrage de référence sur la représentation politique: le principe de justice et d'équité (l'absence ou la sous-représentation politique systématique constituant une faille pour un système démocratique), le rôle de modèle, le renouvellement politique (à travers un style différent) et la défense d'intérêts particuliers (Phillips 1995, 62-63). Quelle qu'en soit la raison, en tant que première femme à occuper la fonction de Premier ministre, Margaret Thatcher n'a pas agi en faveur des femmes dans le monde politique en s'entourant de femmes, conseillères ou ministres.

\section{Un manque d'initiative personnelle}

L'examen des différents gouvernements et Cabinets formés par Margaret Thatcher révèle qu'elle n'a rien fait pour promouvoir davantage de femmes. Une seule a été nommée au sein du Cabinet. Lorsque le journaliste du Guardian Jonathan Steel l'interroge sur son manque d'initiative sur ce sujet, elle cite effectivement le nom de Lady Young, précisant qu'elle a fait partie du Cabinet "pendant longtemps » $^{74}$. En réalité, Lady Young (Janet Mary Baker Young, décédée en 2002) a été membre de droit du Cabinet en tant que leader de la Chambre des Lords, pendant moins de deux ans, de septembre 1981 à juin 1983. Sur les onze années au cours desquelles Margaret Thatcher a été au pouvoir, ce fut la seule nomination d'une femme au sein du Cabinet. Devant le congrès des femmes conservatrices de 1988, elle déclare qu'afin "d'avoir une représentation féminine plus importante au gouvernement, il faut davantage de femmes au Parlement, ce qui signifie qu'un nombre plus important de femmes candidates doivent être sélectionnées, et davantage parmi vous doivent se présenter $\aleph^{75}$. Il ne s'agit donc pas, selon elle, d'une mauvaise volonté de sa part, mais d'un problème de recrutement, d'un vivier insuffisant, les femmes n'étant pas intéressées par la politique, ce qui constitue l'un des arguments traditionnels avancés par ceux et celles qui contestent l'utilité de toute forme de discrimination positive. A aucun moment, elle ne remet en question sa position et elle rejette la faute sur le système politique britannique et ses usages qui contraignent le Premier ministre à choisir ses ministres parmi les membres des deux chambres du Parlement, un choix restreint, à la différence d'autres pays ${ }^{76}$.

A travers certaines références historiques, elle salue pourtant l'adoption du droit de vote pour les femmes en Grande-Bretagne, rappelant que cela ne fait pas si longtemps que les femmes peuvent voter et que le suffrage universel n'était pas en vigueur quand elle est née ${ }^{77}$. Elle rend également hommage à certaines pionnières, comme Lady Astor qui a prouvé qu'" une femme n'avait pas à se montrer masculine pour réussir dans un monde d'hommes ${ }^{78}$ et qui a servi de modèle pour d'autres femmes aux aspirations politiques $^{79}$. Si Margaret Thatcher déplore le nombre peu élevé de femmes parlementaires, en revanche, elle n'indique pas de moyen de l'augmenter. 


\section{Une absence de moyens}

29 Margaret Thatcher ne mentionne aucune solution pour parvenir à une meilleure représentation numérique des femmes au Parlement et elle se refuse à toute mesure de discrimination positive. Elle ne perçoit pas les femmes comme victimes de discrimination et estime que toute nouvelle législation visant à garantir l'égalité des chances est inutile. Lors d'une interview télévisée deux ans après avoir pris ses fonctions, elle déclare que « rien de plus ne peut être fait en changeant la loi » et qu'il y a peu de discrimination, puisque, depuis des années, des femmes parviennent à être élues au Parlement et à devenir ministres ${ }^{80}$. Par ailleurs, la législation garantit déjà l'égalité salariale au Parlement, ce qui n'a pas augmenté le nombre de femmes rétorque-t-elle lors d'une séance de questions au Premier ministre en janvier $1989^{81}$. C'est aux femmes de saisir les chances qui leur sont offertes, de montrer ce qu'elles valent et de faire leurs preuves ${ }^{82}$. En comparant la Grande-Bretagne à certains pays en voie de développement, elle suggère que les femmes britanniques, elles, manquent de motivation : «Une chose m'a toujours frappée : il y a davantage de femmes Premiers ministres dans les pays en voie de développement que dans le monde occidental [...] très étrange que les occasions aient été relativement limitées mais les femmes politiques ont su les saisir $"^{83}$. Un mois avant de démissionner, elle déplore que «les femmes n'osent pas encore se mettre en avant $»^{84}$. Pourtant, selon le Premier ministre, aucune fonction n'est inaccessible aux femmes et elle s'élève contre la ségrégation verticale et la division sexuelle des postes gouvernementaux :

Cela ne s'arrête pas à ce que l'on pourrait qualifier de rôle traditionnel de la femme [...] la Défense est tout aussi importante pour les femmes que pour les hommes [...] les femmes devraient être très douées pour la Finance [...] les femmes devraient s'intéresser à l'Education avec passion [...] les femmes devraient être très compétentes aux Transports, aux Affaires étrangères, à tout un tas de choses ${ }^{85}$.

Elle se base sur son exemple personnel de réussite grâce au travail et se vante de n'avoir bénéficié d'aucune mesure de faveur ${ }^{86}$. Sa vision découle donc, en partie, de son éducation et de son parcours personnel, qu'elle raconte volontiers aux journalistes. Régulièrement, elle affirme ne pas tenir compte de cette spécificité genrée :

Je ne pense pas : je suis une femme Premier ministre, je suis différente. Je pense : je suis Premier ministre ; qu'est-ce que je souhaite faire pour représenter mon pays? Comment vais-je m'y prendre? On ne pense pas en termes de femme Premier ministre, femme ministre ou femme député, on pense : c'est mon métier, comment vais-je atteindre mes objectifs ? Quelle est la meilleure façon de le faire ? ${ }^{87}$

31 Elle affirme que sa féminité n'est pour rien dans son ascension politique, qui n'est due qu'à son travail et à ses compétences. Par ailleurs, si elle est parvenue à atteindre de telles fonctions, rien n'empêche d'autres femmes de faire de même et il n'est nul besoin de mesures spécifiques. Cet avis se trouve partagé par deux autres pionnières politiques, Indira Gandhi et Golda Meir : « toutes trois avaient travaillé dur pour réussir et elles ne voyaient donc pas l'utilité de prendre des mesures qui faciliteraient l'insertion des femmes dans le monde politique »(Steinberg 9) $)^{88}$, ce qui correspond à la catégorie anti-féministe que R. Rowland qualifie de «reine des abeilles »: "Les 'reines des abeilles' ont une idéologie individualiste, sachant qu'elles ont réussi grâce à leurs propres efforts et estimant, par conséquent, que toutes les femmes peuvent le faire " (Rowland 496). ${ }^{89}$ Margaret Thatcher refuse d'ailleurs de s'ériger en modèle, déclarant : «Ce n'est pas une victoire pour les femmes. C'est la victoire d'une personnalité politique ", "Je ne remarque pas que je suis une femme. Je me considère comme 
Premier ministre $»^{90}$. Elle a pourtant dû faire face à des manifestations sexistes et son ascension politique ne s'est pas faite sans provoquer commentaires acerbes et moqueries. Ainsi, le 9 octobre 1959, jour de son entrée au Parlement, le London Evening News titra «La maman de Mark est député maintenant " ${ }^{91}$, et lorsqu'elle pénétra à la Chambre des communes, elle fut accueillie, comme elle le décrit elle-même dans ses mémoires, par « une grande hilarité machiste» et par des boutades du type «Donnenous un baiser, Maggie » (Thatcher 1995, 284) ${ }^{92}$.

Le refus de prendre des mesures qui faciliteraient l'accès des femmes au monde politique explique que, par exemple, lors du scrutin de 1987, les femmes représentaient seulement $14 \%$ de l'ensemble des candidats, avec un pourcentage deux fois inférieur pour le Parti conservateur ( $7,3 \%$ contre $14,6 \%$ pour les travaillistes et $16,6 \%$ pour les Libéraux) (Evans 24). Ce n'est que sous l'influence de Tony Blair dans les années 1990 et de David Cameron dans les années 2000 que de réelles mesures furent adoptées par les partis, comme les listes de candidats composées uniquement de femmes dans certaines circonscriptions.

\section{Conclusion}

Le positionnement de Margaret Thatcher en tant que première femme à la tête de la Grande-Bretagne apparaît comme particulièrement complexe sur la question du genre et des "enjeux féminins", caractérisé par l'ambivalence et l'ambiguïté. Comme le résume Jane Pilcher, "au pouvoir, elle envoya des messages contradictoires et déconcertants aux femmes et à leur sujet " (194) ${ }^{93}$. Son parcours, son comportement, ses politiques et les sentiments qu'elle a suscités révèlent de nombreux paradoxes, et «les féministes elles-mêmes divergent quant aux conséquences principales du Thatchérisme pour les femmes, même si peu peuvent affirmer qu'elles ont été positives ", pour reprendre les termes de Joni Lovendusky (141) ${ }^{94}$. Loin de faire avancer la cause des femmes, nombreuses sont celles qui considèrent que le fait d'avoir une femme à la tête du pays leur a été plus néfaste que bénéfique. L'analyse de cette exception britannique montre que plus qu'une question de sexe, il s'agit surtout d'une personnalité possédant par exemple une capacité de travail et de maîtrise des détails hors du commun. Si John Major, qui lui succéda, a fréquemment été décrit comme présentant un visage plus humain du conservatisme, il possédait également une masculinité peu affirmée, qui contrastait avec son prédécesseur.

L'analyse des déclarations, discours, interviews et politiques de Margaret Thatcher témoigne des limites de la corrélation directe entre les caractéristiques sociologiques, comme le genre, et l'action politique. A défaut d'illustrer le lien direct entre la nature des représentants et le type de politiques mises en place, il semble donc que l'impact de Margaret Thatcher s'assimile davantage à un rôle de modèle et d'émulation, certaines femmes politiques la citant effectivement comme étant à l'origine de leur vocation et ayant démontré qu'il était possible, en Grande-Bretagne, de briser le plafond de verre pour parvenir au sommet du pouvoir politique. Qu'il s'agisse de se comporter comme un représentant d'un sous-groupe de population ou comme n'importe quel autre responsable politique, aucune femme n'est, à ce jour, parvenue à être élue à la tête d'un parti susceptible de former le prochain gouvernement britannique. 


\section{BIBLIOGRAPHIE}

Bashevkin, Sylvia. « Tough Times in Review. The British Women's Movement during the Thatcher Years » Comparative Political Studies 28:4 (January 1996).

Bird, Karen. « Gendering Parliamentary Questions » British Journal of Politics and International Relations 7:3 (2005): 353-370.

Childs, Sarah. Women and British Politics. Descriptive, Substantive and Symbolic Representation. London: Routledge, 2008.

--- « Competing Conceptions of Representation and the Passage of the Sex Discrimination (Election Candidates) Bill » Journal of Legislative Studies 8:3 (2002): 97.

Childs, Sarah, Julie Withey. « Women Representatives Acting for Women: Sex and the Signing of Early Day Motions in the 1997 Parliament » Political Studies 52:3 (2004): 560.

Conservative Party. Conservative Manifesto 1979,

<http://www.politicsresources.net/area/uk/man.htm>

Conservative Party. The Challenge of Our Times 1983,

<http://www.politicsresources.net/area/uk/man.htm>

Conservative Party. The Next Moves Forward 1987,

<http://www.politicsresources.net/area/uk/man.htm>

Cullen, Catherine. Margaret Thatcher. Une Dame de Fer. Paris : Odile Jacob, 1991.

Evans, Eric J. Thatcher and Thatcherism. London: Routledge, 1997.

Hansard Parliamentary Archive, 1979-1990, <www.legislation.gov.uk>

Karam Azza M., Joni Lovenduski (ed.). Women in Parliament: Beyond Numbers. Stockholm: International IDEA, 1998.

Kavanagh, Dennis, Anthony Seldon (eds). The Thatcher Effect. A Decade of Change. Oxford: Oxford University Press, 1989.

Kent, Susan Kingsley. Gender and Power in Britain, 1640-1990. London: Routledge, 1999.

Leruez, Jacques. Le phénomène Thatcher. Paris : Editions Complexe, 1991.

Lovenduski, Joni, Vicky Randall. Contemporary Feminist Politics. Women and Power in Britain. Oxford: Oxford University Press, 1993.

Margaret Thatcher Foundation, 1979-1990, <http://www.margaretthatcher.org>

Minogue, Kenneth, Michael Biddiss. Thatcherism: Personality and Politics. Basingstoke: Macmillan, 1987.

Norris, Pippa. « Women Politicians: Transforming Westminster? » Parliamentary Affairs 49:1 (1996): 91-92.

Norris, Pippa, Joni Lovenduski. « Blair's Babes: Critical Mass Theory, Gender and Legislative Life ", Paper for the Women and Public Policy Program weekly seminar, September 28, 2001, Kennedy school of Government. 
Nunn, Heather. Thatcher, Politics and Fantasy. The Political Culture of Gender and Nation. London: Lawrence \& Wishart, 2002.

Perfect, David. Gender Pay Gaps, Equality and Human Rights Commission, briefing paper 2, 2011.

Phillips, Anne. The Politics of Presence. Oxford: Clarendon, 1995.

Pilcher, Jane. «The Gender Significance of Women in Power. British Women Talking about Margaret Thatcher » The European Journal of Women's Studies 2:4 (1995).

Rivière-De Franco, Karine. «The Parliamentary Behaviour of Women and Men MPs: Equal Status, Similar Practices?» LISA e-journal 12:7 (2014).

Rowland, R. « Women Who do and Women Who don't Join the Women's Movement » In " The Gender Significance of Women in Power. British Women Talking about Margaret Thatcher ", Pilcher, Jane. The European Journal of Women's Studies, 2:4 (1995).

Steinberg, Blema S. Women in Power. The Personalities and Leadership Styles of Indira Gandhi, Golda Meir and Margaret Thatcher. London: McGill-Queen's University Press, 2008.

Thatcher, Margaret. The Downing Street Years. London: Harper Collins, 1993.

---, The Path to Power. London: Harper Collins, 1995.

Thomson, Andrew. Margaret Thatcher: The Woman Within. London: W. H. Allen, 1989.

Trimble, L., J. Arscott. Still Counting: Women in Politics Across Canada. Peterborough: Broadview Press, 2003.

Webster, Wendy. Not a Man to Match Her. The Marketing of a Prime Minister. London: Women's Press, 1990.

Young, Hugo. One of Us. London: Pan Books, 1990.

\section{NOTES}

1. Le suffrage universel est établi en 1928, Representation of the People Act, 1928.

2. <http://www.parliament.uk/get-involved/outreach-and-training/parliament-and-women>, consulté le 23/04/2014.

3. L'Irlande du Nord pour Mo Mowlam (1997), les Affaires étrangères pour Margaret Beckett (2006), et l'Intérieur pour Jacqui Smith (2007).

4. Depuis les années 2000, d'autres partis ont élu des femmes à leur tête: les Verts (Caroline Lucas, 2008-2012, Natalie Bennett, 2012-), les Libéraux Démocrates gallois (Kirsty Williams, 2008) et Plaid Cymru (Leanne Wood, 2012-), les travaillistes écossais (Johann Lamont, 2011-), les conservateurs écossais (Annabel Goldie, 2005-2011, Ruth Davidson, 2011-) et le Parti national écossais (Scottish National Party) (Nicola Sturgeon, 2014-).

5. «It will be years before a woman either leads the party or becomes Prime Minister. I certainly do not expect to see it happening in my time ", <http://www.margaretthatcher.org/document/102369>, consulté le 24/06/2014.

6. Expression utilisée pour la première fois dans un discours à Cardiff le 16 avril 1979, <http:// www.margaretthatcher.org/document/104011>, consulté le 23/06/2014.

7. Cela fut fréquemment le cas pour Geoffrey Howe, ministre des Finances puis des Affaires étrangères, dont le discours de démission au Parlement le 13 novembre 1990 conduit à la démission de Margaret Thatcher. 
8. Ces termes («wet », «dry») datent des années 1940, mais ont été popularisés sous Margaret Thatcher, qui utilisait le terme "timoré» pour désigner les conservateurs de l'aile gauche, représentée notamment par Ian Gilmour, Jim Prior et John Biffen.

9. Majorité de 43 sièges en 1979, 144 en 1983 et 102 en 1987, <Erreur ! Référence de lien hypertexte non valide. consulté le 17/07/2014.

10. Archives de Margaret Thatcher, Margaret Thatcher Foundation, <Erreur! Référence de lien hypertexte non valide. consulté en juin-juillet 2014.

11. Conservative Manifesto 1979, The Challenge of Our Times 1983, The Next Moves Forward 1987, <http://www.politicsresources.net/area/uk/man.htm>, consulté en juin 2014.

12. Archives parlementaires, Hansard Parliamentary Archive, <Erreur! Référence de lien hypertexte non valide. consulté en juin 2014.

13. "But do you think, as a female prime minister, you have women in the back of your mind in terms of policies you make? / Yes, very much so, very much so ", interview, Central TV, 18/06/1986.

14. "What are you doing for women? How are you helping them? / Well, we believe that women help themselves and we are trying to get more and more opportunities for them ", interview, Channel 4, 24/05/1987.

15. " As I indicated, you do not have a policy especially for women. Women now have opportunities we never had before. I would not be here if we did not. I just want more women to take advantage of those opportunities ", conférence de presse, 10/06/1987.

16. «Policies are for all the people. I respectfully point out that we really do not live in wholly separate compartments ", Ibid.

17. Séance de questions au Premier ministre, 27/06/1985.

18. Séance de questions au Premier ministre, 22/06/1989.

19. Séance de questions au Premier ministre, 28/11/1989, 08/03/1990, 13/03/1990.

20. "Last week's Budget marked a milestone for women [...] I'm proud to be a member of a government which has brought in this measure-this overdue measure of justice for women ", discours, 31/03/1990. "Three million married women will pay less as a result of these changes [...] These reforms have promoted women's independence and protected their privacy ", discours, 18/07/1990.

21. 581 Public General Acts, mai 1979-novembre 1990, Hansard Parliamentary Archive, <Erreur! Référence de lien hypertexte non valide. consulté en juin 2014.

22. < http://www.eurofound.europa.eu/emire/UNITED\%20KINGDOM/ SEXDISCRIMINATIONACT1986SDA1986-EN.htm>, consulté le 18/07/2014.

23. " heroic narratives of masculine courage and strength and stoic national pride ».

Il convient néanmoins de garder à l'esprit que le souverain britannique, homme ou femme, reste le chef suprême des armées.

24. "The agenda of Britain's first female Prime minister appeared, ironically, to collide head-on with many feminist priorities ».

25. "The chief silence, the chief absence from Mrs Thatcher's convictions as a self-proclaimed 'conviction politician' concerns gender $»$.

26. "I find that if industry finds a woman who is very very able she is just as likely to be promoted as any other person of ability ", discours, 24/02/1987.

27. En 2007, cette commission fut incorporée à l'Equality and Human Rights Commission, $<$ Erreur ! Référence de lien hypertexte non valide.

28. "I think it would be a great mistake to disband it [EOC] [...]The way prejudice is broken down is by women of the right qualifications, the right personality, coming along and just getting on with the job ", discours, 29/07/1988.

29. "I judge people really rather by their personality, not by whether they are men or women; but whether they have a strong enough personality, whether they are decisive enough, whether they are prepared to face questions and not run away from them; and that is much much more important than the particular sex ", interview, télévision italienne (RAI), 10/03/1986. 
30. "I think the difference is in personality far from the differences between the male and female in the issues that I have to deal with ", interview, magazine Parade, 17/04/1986.

31. "The battle for women's rights has been largely won ", discours, 26/07/1982.

32. "I hated those strident tones that you still hear from some Women's Libbers' », Ibid.

33. "I'm not very keen on it [women's Lib'] [...] No, because I think most of us got to our own position in life without Women's Lib and we got here, not by saying 'you've got to have more women doing so and so' but saying 'look, we've got the qualifications, why shouldn't we have just as much a chance as a man?' And you'll find that so many male bastions were conquered that way, whereas Women's Lib, I think, has been rather strident, concentrated on things which don't really matter and, dare I say it, being rather unfeminine ", interview, Thames TV CBTV, 13/12/1982.

34. "Conservative women are, above all, practical. For example, we don't seek to advance women's rights by insisting that you, Madam Chairman, be addressed as Madam Chairperson, Madam Chair, or, worse still, just plain Chair ", discours, 25/05/1988.

35. "I know that my mother coped with her life and I just think that women have a special capacity to cope. They manage to cope with a job, they manage to cope with home, they manage to cope with bringing up children, they manage to cope where there's any emergency. And I'm no different from any other ", interview, London Weekend Television, 19/07/1984.

36. "I only know that whatever happens I have to take what comes and just cope with it and just get on with it. But then that is what most women do anyway and they do it very well », interview, Channel 4 , 06/11/1989.

37. Interview, CBS 60 Minutes, 15/02/1985.

38. "It is partly, I think, that they prefer getting things done rather than making speeches about it and I notice that when women are in Parliament they are extremely practical about how they can move things forward ", interview, Central TV, 18/06/1986.

39. "On the European Council, really, our French friends were so very very difficult and what they did was so very non-commonsense, and women are very good at common sense and men are not ", interview, ITN News At Ten, 18/02/1988.

40. Interview, TV-AM, 30/12/1988. Discours, 08/17/1989.

41. Interview, Central TV, 18/06/1986.

42. Interview, Channel 4, 24/05/1987.

43. "I can't help reflecting that it's taken a Government headed by a housewife with experience of running a family to balance the books for the first time in twenty years-with a little left over for a rainy day ", discours, 25/05/1988.

44. "I do enjoy working with women [...] when you are dealing with women who are helping you with taking down the speeches, typing them, they are very efficient and their dedication to the job is total ", interview, Channel 4 News, 13/06/1986.

45. Discours, 23/02/1982. Discours, 12/10/1984. Interview, Channel 4, 15/10/1984. Interview, Woman's Own, 16/10/1984. Conférence de presse, 07/12/1984. Conférence de presse, 10/06/1985. Interview, Esportare, 04/09/1986. Interview, Thames TV, 04/06/1987.

46. "However, that rise did not have an impact on the unemployment register because many married women came into jobs », discours, 26/02/1985.

47. " and what is wrong with having a part-time job, many women like a part-time job? [...] do not denigrate part-time work. Many women like part-time work. It suits them. It raises the standard of living of their family and then they go out and spend what they have earned or part of it and then they spend it on other things ", interview, Channel 4 News, 13/06/1986.

48. "What is wrong with working part-time? Many women like it. It suits the pattern of running the house. Often it suits the pattern of being home with the children for some part of the day ", interview, Télévision écossaise, 04/09/1986.

49. Séance de questions au Premier ministre, 14/06/1990. Interview, News of the World, 03/08/1990. 
50. "It is vital that we safeguard the institution of family life, and undoubtedly the mother has the most important role in bringing up the children ", séance de questions au Premier ministre, 23/01/1990.

51. Interview, The Sun, 28/02/1983.

52. «But it is for them to choose ", Ibid.

53. "I beg, I beg, I beg, never put the children second", interview, Thames TV Afternoon Plus, 06/01/1981.

54. " it is for mothers to decide whether they can make full and proper arrangements for their children or whether they should wait until later, when their children are off their hands, to return to work ", séance de questions au Premier ministre, 28/11/1989.

55. Interview, Yorkshire Television Woman to Woman, 02/10/1985.

56. Ibid.

57. «In my case, preparation for the election involved more than politics. I also had to be dressed for the occasion ».

58. « Too many businesses still slimming for survival, and I know how difficult slimming is, don't many women? ", discours, 14/05/1982.

59. "I think male Prime Ministers one day will come back into fashion!», interview, TV-AM, $30 / 12 / 1988$.

60. Discours, 17/02/1982.

61. "we both belong to that small but distinguished band of women Prime Ministers", discours, 16/11/1983.

62. Interview radio, BBC World Service, 03/06/1990.

63. " as the first woman Prime Minister, she is introducing sexist and racist legislation that will make lots of women in my constituency second-class citizens ", séance de questions au Premier ministre, 04/12/1979.

64. Séance de questions au Premier ministre, 30/10/1979.

65. Séance de questions au Premier ministre, 17/02/1983.

66. Séance de questions au Premier ministre, 08/03/1988.

67. "It is a great disappointment to me that in our own Parliament we have only a very small representation of women ", conférence de presse, 15/09/1989. "It has been a great sorrow to me that there are not enough [women] in public life ", interview, Yorkshire TV, 15/09/1987.

68. "we want to double, treble, quadruple, the numbers. Let us make a target first of having a third of the House of Commons consisting of women ", interview, Central TV, 18/06/1986.

69. " until there are more of us both in the House of Commons and right at the top we'll still be too conspicuous merely by being women ", discours, 23/11/1987.

70. «It would also make the rest of us a good deal less conspicuous and we should be normal instead of being looked at as something of a phenomenon", discours, 24/02/1987. Voir aussi discours, 15/11/1986, 01/10/1990.

71. Interview, Newsweek, 04/05/1983. Interview, Central TV, 18/06/1986. Interview, TV-AM Frost on Sunday, 01/09/1990.

72. Interview, The Daily Mail, 16/03/1984.

73. L'argument a également été avancé par Harriet Harman du Parti travailliste, mais pour elle cela ne représente qu'une raison parmi d'autres.

74. « Lady Young was in my Cabinet for a considerable time », conférence de presse, 12/09/1986.

75. «But if we are to have more women in Government, we must have more women in Parliament. And that means you must select more women candidates-and more of you must put yourselves forward", discours, 25/05/1988.

76. Conférence de presse, 12/09/1986.

77. Discours, 26/11/1984. Discours, 25/05/1988. 
78. «It was a Conservative, Nancy Astor, who was the first woman to take a seat in Parliament and who did so with great style and confidence-proving that a woman does not have to be masculine to succeed in a man's world", discours, 25/05/1988.

79. Séance de questions au Premier ministre, 30/11/1989.

80. "I'm absolutely satisfied there is nothing more you can do by changing the law to do away with either sex discrimination or racial discrimination. After all I don't think there's been a great deal of discrimination against women, for years, you've been able to come into Parliament, you've been able to be Ministers, you've been able to do many, many things which not many women have taken the opportunity to do ", interview, Thames TV Afternoon Plus, 06/01/1981.

81. Séance de questions au Premier ministre, 19/01/1989.

82. Interview, Thames TV Afternoon Plus, 06/01/1981.

83. "One thing that has always struck me is that there have been more women Prime Ministers in the developing world than in the Western World [...] Very strange that the opportunities for women were comparatively small in developing countries but the opportunities and value of women politicians have been seized upon in developing countries, greatly to the advantage of women and to those developing countries ", discours, 01/10/1990.

84. "women still seem to be a little bit shy about coming forward ", Ibid.

85. "It does not stop at what one might call the traditional woman's role. I do want to make this clear: defence is just as important to women as it is to men [...] Women should be extremely good at finance [...] Women should be passionately interested in education [...] Women should be very good at transport, very good at foreign affairs, very good at the whole range of things ", Ibid.

86. Discours, 18/07/1990.

87. "You do not start to think: "Now look, I am a woman prime minister, I am different." You think: "I am a Prime Minister. What do I want to do as representing my country. How am I going to get it through?" You do not think in terms of being a woman prime minister or a woman minister or a woman member of Parliament. You think in terms of: "This is my job, how am I going to achieve my objectives? How best can I do it? What is the best way to do it? ", Interview, Central TV, 18/06/1986.

88. " all three women believed that their accomplishments were based on hard work and thus saw no reason for actions to promote women in politics".

89. "Queen bees have an individualistic ideology, knowing that they have achieved success because of their own efforts and assuming, therefore, that any woman can ».

90. "It's not a victory for women. It's a victory for someone in politics", conférence de presse, 11/02/1975, "I don't notice I'm a woman. I regard myself as Prime minister », interview, The Daily Mirror, 01/03/1980.

91. "Mark's Mummy is an MP Now».

92. " much male chauvinist hilarity ", « Give us a kiss, Maggie ».

93. " contradictory and puzzling messages she gave to and about women while she was in office».

94. "Feminists themselves are in disagreement as to the main consequences of Thatcherism for women, though few would argue that they have been good".

\section{RÉSUMÉS}

Cet article se propose d'analyser le positionnement et les politiques de Margaret Thatcher - la première, et, à ce jour, l'unique femme à avoir été Premier ministre en Grande-Bretagne - sous 
un angle spécifique, celui des enjeux traditionnellement qualifiés de "féminins ». En se fondant sur les théories de la représentation et à travers l'analyse de l'ensemble de ses déclarations, discours et interviews lors de ses onze années au pouvoir, il vise à évaluer l'intérêt qu'elle a porté à ces questions ainsi que son impact global sur la population féminine britannique.

This article focuses on Margaret Thatcher - the first and only female Prime Minister in Britain from a specific point of view, the topics which are traditionally referred to as " women's issues ». Using the theories of representation and analyzing all her statements, speeches and interviews over her eleven years in power, it aims at assessing her interest for that subject as well as her general impact on British women's lives.

\section{INDEX}

Keywords : Margaret Thatcher, politics, Parliament, women, representation, women's issues, feminism

Mots-clés : Margaret Thatcher, politique, Parlement britannique, femme, représentation, enjeux féminins, féminisme

\section{AUTEURS}

\section{KARINE RIVIÈRE-DE FRANCO}

Maître de conférences

Université d'Orléans

karine.riviere-de-franco@univ-orleans.fr 